\title{
FIGO Stage IIIA
}

National Cancer Institute

\section{Source}

National Cancer Institute. FlGO Stage IIIA. NCI Thesaurus. Code C96256.

A FIGO stage term that applies to gynecologic cancers. For cervical cancer, it refers to cancer that involves the lower third of vagina and there is no extension to the pelvic wall; for endometrial cancer, it refers to cancer with involvement of the serosa and/or the adnexa. 